\title{
Pressure and Shear Stress in Trabecular Bone Marrow During Whole Bone Loading
}

Thomas A. Metzger ${ }^{1}$, Stephen A. Schwaner ${ }^{2}$, Anthony J. LaNeve ${ }^{2}$, Tyler C. Kreipke ${ }^{1}$, Glen L. Niebur $^{1}$

${ }^{1}$ Tissue Mechanics Laboratory, Bioengineering Graduate Program, University of Notre Dame ${ }^{2}$ Dept. of Aerospace and Mechanical Engineering, University of Notre Dame

May 9, 2015

Submitted to: Journal of Biomechanics

Running Title: Bone marrow mechanical environment

Word Count: 3145

Address Correspondence to:

Glen L. Niebur

147 Multidisciplinary Research Building

Notre Dame, IN 46556

Phone: +1-574-631-3327

Fax: +1-574-631-2174

e-mail: gniebur@nd.edu 


\section{Abstract}

2 Skeletal adaptation to mechanical loading is controlled by mechanobiological signaling.

3 Osteocytes are highly responsive to applied strains, and are the key mechanosensory cells in

4 bone. However, many cells residing in the marrow also respond to mechanical cues such as

5 hydrostatic pressure and shear stress, and hence could play a role in skeletal adaptation.

6 Trabecular bone encapsulates marrow, forming a poroelastic solid. According to the mechanical

7 theory, deformation of the pores induces motion in the fluid-like marrow, resulting in pressure

8 and velocity gradients. The latter results in shear stress acting between the components of the

9 marrow. To characterize the mechanical environment of trabecular bone marrow in situ, pore

10 pressure within the trabecular compartment of whole porcine femurs was measured with

11 miniature pressure transducers during stress-relaxation and cyclic loading. Pressure gradients

12 ranging from 0.013 to $0.46 \mathrm{kPa} / \mathrm{mm}$ were measured during loading. This range was consistent

13 with calculated pressure gradients from continuum scale poroelastic models with the same

14 permeability. Micro-scale computational fluid dynamics models created from computed

15 tomography images were used to calculate the micromechanical stress in the marrow using the

16 measured pressure differentials as boundary conditions. The volume averaged shear stress in the

17 marrow ranged from 1.67 to $24.55 \mathrm{~Pa}$ during cyclic loading, which exceeds the

18 mechanostimulatory threshold for mesenchymal lineage cells. Thus, the loading of bone through

19 activities of daily living may be an essential component of bone marrow health and

20 mechanobiology. Additional studies of cell-level interactions during loading in healthy and

21 disease conditions will provide further incite into marrow mechanobiology. 


\section{Introduction}

24 Skeletal adaptation to mechanical loads is controlled by cells that sense the local and

25 spatially averaged deformation of the tissue, and express signaling molecules to surrounding

26 cells that affect the addition or removal of bone tissue. Through this process, bone mineral

27 density decreases during extended periods of inactivity, such as bed rest (LeBlanc et al., 1990)

28 and space flight (LeBlanc et al., 2000), and increases when frequent load-unload cycles are

29 applied through, for example, intense physical activity (Courteix et al., 1998; Tournaire et al.,

30 2007). Osteocyte mechanotransduction is the primary controller of bone adaptation and repair

31 (Bonewald, 2006; Schaffler et al., 2014; You et al., 2008). However, cells in the marrow are also

32 mechanically sensitive (Luu et al., 2009; Qin, 2003; Soves et al., 2014). Indeed, mechanical

33 loading of mouse limbs in vivo induces mechanical signaling between marrow cells (Soves et al.,

34 2014) and increased proliferation and osteogenic gene expression in marrow stromal cells (Li et

35 al., 2004; Mantila Roosa et al., 2011; Ozcivici et al., 2010).

37 of long and axial bones, and in the pore spaces of trabecular bone (Tavassoli and Yoffey, 1983).

38 Bone marrow is the niche for multipotent mesenchymal stromal cells (MSCs), which are

39 progenitors for connective tissue cells capable of differentiating into osteogenic, chondrogenic,

40 and adipogenic lineages (Rosen and Bouxsein, 2006), and hematopoietic stem cells from which 
41 both blood and immune cells derive, including macrophages such as osteoclasts. Marrow cells

42 respond to mechanical stimuli. For example, steady and oscillatory shear stress both affect MSC

43 differentiation to the osteogenic lineages (Castillo and Jacobs, 2010; Li et al., 2004), as well as

44 nitric oxide (NO) and prostaglandin production in marrow-derived pre-osteoclast (McAllister et 45 al., 2000).

46 Loading of whole bones increases the pressure in the bone marrow. In human femurs

47 tested in vitro, the pressure reached 1 to $2 \mathrm{kPa}$ during physiologic loading (Downey et al., 1988).

48 The pressure was higher in the head than the neck, and increased with loading rate. Impact

49 loading of ovine tibia resulted in pressures as high as $35 \mathrm{kPa}$ in the trabecular pore space (Bryant,

50 1983). These pressures may be too low to provide substantial load-bearing effects (Arramon and

51 Cowin, 1997; Bryant, 1983; Kafka, 1983; Ochoa et al., 1991), and are below previously

52 measured limits for mechanotransduction in MSCs (Castillo and Jacobs, 2010) and

53 hematopoietic lineage cells, such as pre-osteoclasts (Kwon et al., 2012). However, the pore

54 deformation forces motion of the fluid-like marrow, which is resisted by shear stress between the

55 marrow components. Since marrow is composed primarily of cells, this shear stress would be

56 sustained by intercellular interactions.

57 We hypothesized that the shear stress in the trabecular bone marrow during loading

58 reaches a sufficient magnitude to affect cell mechanobiology. As such, the goal of this study was 
59 to measure pressure and pressure gradients in whole bones under quasi-physiologic loading and

60 determine the associated marrow kinetics and kinematics. Specifically, we 1) measured

61 pressures at four locations in the neck of fresh porcine femurs during stress relaxation and cyclic

62 loading; 2) parametrically investigated the interaction between strain rate and pore pressure

63 gradients using continuum poroelastic models; and 3) calculated the fluid flow using high-

64 resolution finite element models to determine the associated shear stress distributions and

65 velocity gradients in the marrow.

66 Methods

67 Nine freshly harvested porcine femurs were taken from animals 6-8 months of age

68 (approximately $115 \mathrm{~kg}$ ). The bones were obtained from a local abattoir (Martin's Custom

69 Butchering, Wakarusa, IN) at the time of slaughter. Testing was completed within 8 hours post-

70 mortem, as bone marrow properties are highly sensitive to storage (Metzger et al., 2014).

71 Miniature pressure transducers (Model 060 (1.5 x $0.3 \mathrm{~mm})$, Precision Measurements Inc., Ann

72 Arbor, MI) were used to measure the pore pressure in situ during cyclic and stress-relaxation

73 loading. Four holes approximately $5 \mathrm{~mm}$ apart and $150 \mathrm{~mm}$ deep were prepared along the

74 curvature of the femoral neck using a 2.38-mm drill bit (Fig. 1A). The pressure transducers were

75 inserted through cannulae (Harvard Apparatus, Holliston, MA), which were press-fit

76 approximately $147 \mathrm{~mm}$ into the holes, with the transducers approximately $2 \mathrm{~mm}$ exposed from 
77 the cannulae (Fig. 1A-inset). The cannulae were subsequently backfilled with tap water, and

78 sealed with epoxy to avoid air bubbles (Fig. 1A). Two strain gauge rosettes were fixed to the

79 anterior and posterior aspects of the lesser trochanter (Fig. 1A). The surface of the bone was

80 sanded to remove the periosteum and degreased with ethanol prior to strain gauge attachment

81 with cyanoacrylate glue, with the gauge leads sealed with polyurethane (Viceconti et al., 1992).

82 A custom fixture was designed to maintain the temperature and hydration of the bone

83 during testing (Fig. 1B). The bones were tested while submerged in water, and a circulating bath

84 was used to maintain the water temperature at $37^{\circ} \mathrm{C}$, as bone marrow viscosity depends strongly

85 on temperature (Bryant et al., 1989; Metzger et al., 2014). The distal femur was potted in

86 PMMA and fixed to the bottom of the water tank. Compressive loading was applied to the

87 femoral head through a hemispherical loading platen.

88 Three bones were subjected to stress-relaxation loading. A $2 \mathrm{~mm}$ displacement was

89 applied to the femoral head at a rate of approximately $0.5 \mathrm{~mm} / \mathrm{s}$ and held for 600 seconds. The

90 remaining six bones were subjected to sinusoidal cyclic compression from approximately 600 to

$912000 \mathrm{~N}$ at $1 \mathrm{~Hz}$. Pressure, strain, and load data were monitored at $100 \mathrm{~Hz}$ (LabVIEW, National

92 Instruments, Austin, TX). The data were filtered using a $5 \mathrm{~Hz}$ third-order Butterworth filter.

93 Pressure differentials between the three pairs of adjacent transducers in each bone specimen were

94 calculated for the first full load cycle based on the 3-D transducer spacing determined from $\mu$ - 
95 computed tomography ( $\mu$-CT) imaging as described subsequently. Average pressure gradients

96 were determined by taking the absolute value of the pressure gradients over the first 5 sec of

97 loading for the stress-relaxation specimens and over the first, full $1 \mathrm{sec}$ cycle of the cyclically

98 loaded specimens (Table 1).

99 Following testing, the bone specimens were imaged twice with computed tomography.

100 The first image was taken at $80 \mu \mathrm{m}$ to measure the transducer spacing (Fig. 2A). The femurs

101 were then sectioned and $20 \mu \mathrm{m}$ resolution images of the metaphysis were obtained at $70 \mathrm{kVp}$ and

$102114 \mathrm{~mA}$ (Scanco $\mu \mathrm{CT}-80$, Brüttisellen, Switzerland) (Fig. 2B). The high-resolution $\mu$-CT

103 images of the trabecular bone between each pair of pressure sensors were used to create a finite

104 element model of the bone marrow within a $4 \mathrm{~mm}$ cubic region (Birmingham et al., 2013;

105 Coughlin and Niebur, 2012). Briefly, images were Gaussian filtered. The marrow regions were

106 converted to tetrahedral finite element meshes having from $3,669,000$ to $6,988,000$ tetrahedral

107 elements using the marching cubes algorithm in Visualization Toolkit (VTK, Kitware, Clifton

108 Park, NY). A $200 \mu \mathrm{m}$ layer of fluid was added around the entire boundary of the region of

109 interest to allow pressure boundary conditions to be applied uniformly to the superior and

110 inferior faces. A no-slip condition was applied to this layer of fluid on the perpendicular faces

111 of the models (Fig 2C-inset). 

Continuum poroelastic models were created to estimate the trabecular strain rates

113 required to induce the pressure gradients found experimentally. Five 4 x 4 x $4 \mathrm{~mm}$ cubes were

114 modeled with one thousand hexahedral elements (ADINA, Watertown, MA) (Fig. 3). The bone

115 was assigned a uniform isotropic modulus of $500 \mathrm{MPa}$ and Poisson's ratio of 0.3 (Keaveny et al.,

116 2001). Orthotropic permeability tensors were computed from micro-scale models of the

117 marrow. Briefly, finite element models of the marrow space were analyzed with a Newtonian

118 fluid property and a viscosity of $0.5 \mathrm{~Pa} \bullet \mathrm{s}$. A pressure of $80 \mathrm{~Pa}$ was applied to one face with a 0

$119 \mathrm{~Pa}$ pressure assigned to the opposite face (Fig. 3A). The remaining faces had free-flow

120 boundary conditions. Three such models, with pressure applied along three orthogonal

121 directions, allowed the three columns of the Darcy permeability tensor to be calculated assuming

122 a linear relationship between the pressure gradient and flow rate (Fig. 3B). The calculated

123 permeability (Table 2) was consistent with those previously found experimentally (Arramon and

124 Nauman, 2001; Nauman et al., 1999). The permeability was applied assuming a marrow

125 viscosity of $85 \mathrm{~Pa} \cdot \mathrm{s}$.

126 The continuum models were analyzed at five strain rates from $0.1 \% \mathrm{~s}^{-1}$ to $1.0 \% \mathrm{~s}^{-1}$ in

127 order to cover the range of strains potentially experienced within the trabecular bone of the

128 femoral neck. Zero pore pressure was prescribed on the proximal face of the cube, while fluid

129 flux was constrained on the distal and lateral faces (Fig. 3C). To understand the role of the 
130 constrained flow conditions on the pore pressure, a second solution for each model was obtained

131 with zero pore pressure on the loaded face as well as the lateral faces, allowing the fluid to flow

132 freely through all but the distal face (Fig. 3D). Pore pressure was analyzed at the distal face of

133 the loading for both flow conditions to determine the resulting pressure gradient as a function of

134 loading rate.

135 Computational fluid dynamics models were used to calculate the shear stress distribution

136 of the bone marrow within the trabecular pore space of each sample to obtain a detailed

137 understanding of the effects on the marrow mechanical environment. In these models, the fluid

138 elements were assigned non-Newtonian fluid properties with a density of $0.95 \mathrm{~g} / \mathrm{cm}^{3}$. The

139 viscosity was assigned a power-law relationship of $\eta=85.5(\dot{\gamma})^{-1}$, where $\eta$ is viscosity $(\mathrm{Pa} \bullet \mathrm{s})$

140 and $\dot{\gamma}$ is the shear rate $\left(\mathrm{s}^{-1}\right)$. For numerical stability, the model incorporates a maximum

141 viscosity, which was set to $100 \mathrm{~Pa} \bullet \mathrm{s}$. We varied this maximum viscosity from 47.5 to $1500 \mathrm{~Pa} \bullet \mathrm{s}$

142 to determine the sensitivity. This model was based on our previous measurements in fresh

143 porcine bone marrow using a parallel plate rheometer (Metzger et al., 2014). Because the

144 exponent in the reslationship is -1, this constitutive law has similar characteristics to Herschel-

145 Bulkley model with a yield stress of $85 \mathrm{~Pa}$ and dynamic viscosity of 0 (Metzger et al., 2014).

146 The marrow was assumed to be a homogenous fluid (Bryant et al., 1989; Coughlin and Niebur,

147 2012; Dickerson et al., 2007; Metzger et al., 2015; Ochoa and Hillberry, 1992). The bone- 
148 marrow interface was assigned a rigid no-slip boundary condition, assuming that the adjacent

149 cells were attached to the bone matrix by integrins (Birmingham et al., 2013; Coughlin and

150 Niebur, 2012; Dickerson et al., 2007; Metzger et al., 2015). The experimentally measured time-

151 dependent pressure differentials were prescribed to the proximal face of the bone models and

152 zero pressure was prescribed to the distal face. Fluid flow into and out of the perpendicular faces

153 was constrained (Fig. 2C).

154 A transient, dynamic solution was performed using ADINA-F® (Watertown, MA). The

155 spatial distribution of shear stress within the marrow cavity was analyzed to describe the results.

156 A $3.4 \times 3.4 \times 3.4 \mathrm{~mm}^{3}$ sub-region in the center of the sample was selected for post-processing to

157 avoid artifacts due to boundary conditions. The volumetric mean shear stress and velocity in the

158 marrow were calculated using volume integration in Paraview (Paraview, Kitware, Clifton Park,

$159 \mathrm{NY}$ ). The volumetric averages were further time-averaged at $10 \mathrm{~Hz}$ over the first $1 \mathrm{sec}$ load

160 cycle for cyclic specimens and $10 \mathrm{~Hz}$ over the first $5 \mathrm{sec}$ of loading for stress-relaxation

161 specimens.

162 Results

163 Trabecular pore pressure increased during stress-relaxation loading and dissipated during

164 stress-relaxation (Fig. 4A). On average, the peak pressure was $1.7 \pm 1.3 \mathrm{kPa}(\mathrm{mean} \pm \mathrm{S}$. D.) and

165 ranged from 0.07 to $5 \mathrm{kPa}$. The mean pressure gradient was $0.125 \pm 0.11 \mathrm{kPa} / \mathrm{mm}$ during the 
166 first $5 \mathrm{~s}$ of loading across all the samples. The pressure varied with the load during cyclic

167 loading (Fig. 4B). The peak pressures reached as high as $2.5 \mathrm{kPa}$. The mean pressure gradient

168 was $0.17 \pm 0.11 \mathrm{kPa} / \mathrm{mm}$ during cyclic loading averaged over 1 cycle. The pore pressure

169 gradients did not depend on trabecular bone volume fraction (BV/TV) between adjacent

170 transducers (Fig. 5). The strain rate on the cortical surface was approximately $0.02 \% \mathrm{~s}^{-1} \mathrm{during}$

171 stress-relaxation and $0.12 \% \mathrm{~s}^{-1}$ during cyclic loading.

172 Parametric analysis using poroelastic models indicated that the measured pressure

173 gradients were consistent with deformation-driven fluid motion. The constrained flow model

174 developed pressure gradients from $0.029 \pm 0.027 \mathrm{kPa} / \mathrm{mm}$ when loaded at a rate of $0.1 \% \mathrm{~s}^{-1}$ to

$1750.302 \pm 0.272 \mathrm{kPa} / \mathrm{mm}$ at $1.0 \% \mathrm{~s}^{-1}$ (Fig. 6A). The free-flow model developed pressure gradients

176 an order of magnitude lower than the constrained flow model under similar loading conditions

177 (Fig. 6B).

178 When the measured pressure gradients were applied across the detailed marrow models,

179 the volumetric mean marrow shear stress was $7.1 \pm 6.2 \mathrm{~Pa}$ during stress-relaxation (Fig. 7A) and

$1809.6 \pm 6.9 \mathrm{~Pa}$ when averaged over the 1-second cycle and across all regions analyzed (Fig. 7B).

181 The volumetric mean shear stress increased with the measured pressure differential during both

182 stress-relaxation (Fig. 7A, $\left.\mathrm{R}^{2}=0.73, p<0.005\right)$ and cyclic loading (Fig. 7B, $\left.\mathrm{R}^{2}=0.23, p<0.05\right)$. 
184 across the stress-relaxation models over the first second of loading, and was positively correlated

185 to the measured pressure gradient $(p<0.005)$. During cyclic loading, the volumetric mean

186 marrow velocity averaged $0.01 \pm 0.008 \mathrm{~mm} / \mathrm{s}$ for the six samples over the first cycle, but was not

187 correlated to measured pressure gradient $(p>0.2)$. The peak volumetric mean velocity gradient

188 magnitude within each sample during the first second of stress-relaxation averaged $0.07 \pm$

$1890.06 \mathrm{~s}^{-1}$ across the three samples and $0.11 \pm 0.08 \mathrm{~s}^{-1}$ during the first full cycle across the six

190 cyclically loaded specimens.

191 The average marrow shear stress was not sensitive to the maximum marrow viscosity in

192 the models, as sufficient shear stress must develop in the marrow to resist the applied pressure

193 gradient. Rather, the velocity gradients, and hence the maximum velocity of the marrow are

194 altered by changing the viscosity in the models. When the maximum viscosity in the power-law

195 model was set to $47.5 \mathrm{~Pa} \bullet \mathrm{s}$, the marrow velocity magnitude increased from 0.0194 to 0.0456

$196 \mathrm{~mm} / \mathrm{s}$, while a higher limit of $1500 \mathrm{~Pa} \bullet \mathrm{s}$ decreased the average velocity to $0.0013 \mathrm{~mm} / \mathrm{s}$.

\section{Discussion}

198 While osteocytes are the primary mechanosensors in bone, mechanical stimulation in the

199 marrow cell population may also play an important role in the adaptive response to skeletal

200 loads. Using complementary experiments, imaging, and computational models, this study 
201 demonstrates that deformation of whole bones induces motion, and consequently shear stress, in

202 the bone marrow that could induce a mechanobiological response. The measured pressure was

203 small relative to the applied loads and to the reported mechanobiological response threshold.

204 However, when the pressure gradients were applied in micromechanical fluid dynamics models,

205 the shear stress exceeded the reported threshold levels for mechanobiological response of a

206 number of cell lineages (Table 3). As such, the intercellular motion and deformation of the cells

207 in the marrow space could stimulate mechanobiological pathways in skeletal progenitors directly

208 (Mantila Roosa et al., 2011) or through paracrine signaling from surrounding cells in the marrow

209 (Soves et al., 2014). This mechanobiological signaling could play a role in bone marrow

210 adaptation with aging (Rosen and Bouxsein, 2006), which would have a subsequent effect on

211 bone adaptation, or it could directly affect bone formation (Birmingham et al., 2014; Webster et

212 al., 2015).

213 The in situ micromechanical environment of bone marrow has not been characterized

214 previously. As such, the principle strength of this study was quantification of the marrow

215 micromechanical environment during physiologic loading. In particular, this study quantified

216 the motion and shear stress in trabecular bone marrow within real trabecular pore geometries

217 driven by measured pressure gradients. Although direct measurement of these quantities in

218 whole, intact femurs is not possible with present technology, indirect quantification was achieved 
219 by combining experiments with computational models. While poroelasticity based on Darcy

220 permeability provides a macroscopic view of marrow mechanics, it can only provide a volume-

221 averaged estimate of the fluid environment based on assumed pore geometry and orientation

222 (Arramon and Nauman, 2001; Kohles et al., 2001). The use of micro-scale models provides

223 additional insight into the distribution of shear stress within the pore space. Taken together, a

224 complementary experimental and numerical approach such as the one used here, combining

225 measured pressure and trabecular geometry provided a strategy to assess the marrow mechanics.

226 Computational models at a continuum scale offered insights into the role of strain rate on the

227 measured pressure gradients, while micromechanical models allowed direct computation of the

228 shear stress within the marrow.

229 Although this study provides new insights into the biomechanics of trabecular bone

230 marrow during whole bone loading, several limitations should be considered when interpreting

231 the data. The primary limitation is the use of porcine femora, which differ from human bones in

232 geometry and loading. Porcine bones were used because it was possible to perform the

233 experiments within a short time post mortem, which is essential because of the rapid changes in

234 bone marrow properties during storage (Metzger et al., 2014). For similar reasons, the applied

235 loading was simplified, with no muscle loading on the greater trochanter, which would have

236 required inserting screws and breaching the cortical shell (Ochoa et al., 1997). However, the 
237 study validates the concept of pressure gradients in the trabecular bone, and the methods could

238 be used to extract marrow mechanics from previous or future experiments in human bone under

239 various loading conditions based on multi-scale poroelastic models. A second limitation is the

240 assumption that bone marrow is a homogenous fluid. Bone marrow might be more appropriately

241 characterized as a colloidal suspension of cells. In the continuum poroelastic models, the

242 relationship between pressure gradient and fluid motion is assumed to be linear, which is

243 reasonable for small ranges of fluid velocity (Metzger et al., 2015). In the micro-scale models

244 we modeled bone marrow as a non-Newtonian fluid, as is common with colloidal suspensions of

245 high concentrations (Rutgers, 1962). Capturing the true behavior of the marrow would require

246 multi-scale modeling that incorporates cell-cell adhesion, cell-fluid interaction and multiple cell

247 geometries (Forgacs, 1995; Preziosi et al., 2010; Vaughan et al., 2015).

248 The role of marrow mechanobiology in diseases remains an open question. A number of

249 diseases could affect or be affected by marrow mechanical properties, including osteoporosis

250 (Justesen et al., 2001), diabetes, cancer metastasis (Lynch et al., 2013), and marrow aplasia

251 (Naveiras et al., 2009), where the cell population may be altered. However, we are not aware of

252 any measurements of altered marrow mechanical properties due to diseases. These same

253 diseases are often accompanied by changes in the trabecular bone volume fraction, which affects

254 the permeability, the stiffness, and the resulting strains. Hence, the cumulative effects on bone 
255 marrow shear stress are not easily predictable, as our data for pressure gradient vs. permeability

256 demonstrate (Fig. 6). Higher loading rates, or potentially inertial loading regimes, are the only

257 certain method to increase marrow shear stress in a given disease condition, and these have

258 shown some positive effects in experimental (Judex et al., 2007; Webster et al., 2015; Xie et al.,

259 2006) and clinical studies (Gilsanz et al., 2006).

260 The results complement previous reports on the pressure developed within trabecular

261 bone marrow during whole bone loading. The pressure in proximal human femurs averaged 3.3

$262 \mathrm{kPa}$ when loaded to $1200 \mathrm{~N}$ at $1.0 \mathrm{~s}^{-1}$ and depended on strain rate (Downey et al., 1988).

263 Interestingly, similar pressure increases were measured in an in vivo study in the rat femur during

264 muscle activation (Kumar et al., 1979). The pressure also varied throughout the metaphysis of

265 the ovine tibia during impact loading (Bryant, 1983), but the magnitude was much higher,

266 consistent with the rate dependence of poroelastic materials.

267 Increased bone marrow pressure increases bone formation, although the underlying

268 mechanobiology is not known. Dynamic compression of mouse tibiae induced pressure

269 increases of about $2 \mathrm{kPa}$, and were associated with new bone formation (Hu et al., 2012).

270 Increased intraosseous pressure in a similar range caused by venous ligation also resulted in

271 metaphyseal bone formation in goats (Welch et al., 1993). In rats (Bergula et al., 1999) and mice

272 (Stevens et al., 2006a) increased marrow pressure due to venous ligation, also in the 1 to $2 \mathrm{kPa}$ 
273 range, induced bone formation only in the case of unloaded limbs, suggesting venous ligation

274 compensates for normal marrow pressurization due to mechanical loading. The bone formation

275 was related to increased fluid flow from the marrow into the bone and depended on COX-2 and

276 NOS-3 gene expression (Bergula et al., 1999; Stevens et al., 2006b). The effects of such osseous

277 hypertension appear to depend on non-physiologic pressures. However, the pressures we

278 measured were of similar magnitude and could play some role in normal bone or bone marrow

279 maintenance.

280 The combination of the periosteal strain measurements and poroelastic modeling

281 provided support for the measured pressure gradients found experimentally. The measured

282 periosteal bone strains were approximately $2 \%$ higher than those found in in vivo human femurs

283 during physiologic loading (Aamodt et al., 1997). Previous computational models found the

284 strain in the trabecular bone of the human femoral neck was approximately $0.3 \%$ for similar

285 levels of cortical bone strain (Keyak et al., 1993; Keyak et al., 1990; Lotz et al., 1991; Lotz et al.,

286 1990). Using this as an estimate of the trabecular bone strain over a $1 \mathrm{~s}$ period in these

287 experiments, $2 / 3$ of the experimentally measured pressure gradients fell within the range

288 calculated from the continuum poroelastic models with constrained flow, while the

289 unconstrained boundary conditions under-predicted the measured pressure gradients for most

290 samples (Fig. 6). As such, the constrained flow boundary condition is an upper bound on the 
291 pressure gradient, while the free flow is a lower bound. The constrained case provided a better

292 estimate, which led us to apply a nearly constrained flow condition in the micro-scale models.

293 The use of multiple modeling scales provides a powerful means for investigating marrow

294 mechanics. Fluid-structure interaction models using the same marrow properties (Metzger et al.,

295 2014) resulted in similar pressure gradients to those found in the constrained flow poroelastic

296 models when a strain rate of $0.1 \% \mathrm{~s}^{-1}$ was applied (Metzger et al., 2015). This suggests that

297 poroelastic models can be used to determine trabecular pore pressure and pressure gradients,

298 while micro-scale models enable spatial quantification of shear stress.

299 The concept of hydraulic stiffening and strengthening of whole bone has been

300 controversial (Arramon and Cowin, 1997; Bryant, 1983; Kafka, 1983; Ochoa et al., 1991). In

301 contrast, the inverse question of the role of poroelasticity in marrow mechanobiology has

302 received little attention. Our results demonstrate that interaction between the trabecular bone and

303 marrow generates significant cellular-level motion eliciting mechanical stress and strain within

304 the marrow and its cellular constituents. As such, we propose that the role of bone marrow in

305 resisting mechanical loads during activities of daily living is secondary to the effects these loads

306 play in normal bone marrow mechanobiology, which in turn is essential to human health.

307 Acknowledgements: 
308 This research was supported by the U.S. National Science Foundation CMMI 1100207 and 3091435467.

310

311

312 


\section{References Cited}

314 Aamodt, A., Lund-Larsen, J., Eine, J., Andersen, E., Benum, P., Husby, O.S., 1997. In vivo measurements show tensile axial strain in the proximal lateral aspect of the human femur. J. Orthop. Res. 15, 927-931.

Arramon, Y.P., Cowin, S.C., 1997. Hydraulic stiffening of cancellous bone. Forma 12, 209-221.

Arramon, Y.P., Nauman, E.A., 2001. The intrinsic permeability of cancellous bone, in: Cowin, S.C. (Ed.), The Bone Mechanics Handbook. CRC, New York.

Bergula, A.P., Huang, W., Frangos, J.A., 1999. Femoral vein ligation increases bone mass in the hindlimb suspended rat. Bone 24, 171-177.

Birmingham, E., Grogan, J.A., Niebur, G.L., McNamara, L.M., McHugh, P.E., 2013. Computational modelling of the mechanics of trabecular bone and marrow using fluid structure interaction techniques. Ann. Biomed. Eng. 41, 814-826.

Birmingham, E.C., Kreipke, T.C., Dolan, E.B., Coughlin, T.R., Owens, P., McNamara, L.M., Niebur, G.L., McHugh, P.E., 2014. Mechanical stimulation of bone marrow in situ induces bone formation in trabecular explants. Ann. Biomed. Eng. in press,.

Bonewald, L.F., 2006. Mechanosensation and Transduction in Osteocytes. Bonekey Osteovision 3, 7-15.

Bryant, J., David, T., Gaskell, P.H., King, S., Lond, G., 1989. Rheology of bovine bone marrow. Proc Inst Mech Eng H.

Bryant, J.D., 1983. The effect of impact on the marrow pressure of long bones in vitro. J. Biomech. 16, 659-665.

Castillo, A.B., Jacobs, C.R., 2010. Mesenchymal Stem Cell Mechanobiology. Current osteoporosis reports 8, 98-104.

Coughlin, T.R., Niebur, G.L., 2012. Fluid Shear Stress in Trabecular Bone Marrow due to LowMagnitude High-Frequency Vibration. J. Biomech. 45, 2222-2229.

Courteix, D., Lespessailles, E., Peres, S.L., Obert, P., Germain, P., Benhamou, C.L., 1998. Effect of physical training on bone mineral density in prepubertal girls: a comparative study between impact-loading and non-impact-loading sports. Osteoporos. Int. 8, 152-158.

Cui, L., Li, X.T., Zhang, D., 2012. Effect of fluid flow-induced shear stress on osteoclast formation induced by osteocyte. Zhongguo yi xue ke xue yuan xue bao. Acta Academiae Medicinae Sinicae 34, 207-211.

Dickerson, D.A., Sander, E.A., Nauman, E.A., 2007. Modeling the mechanical consequences of vibratory loading in the vertebral body: microscale effects. Biomechanics And Modeling In Mechanobiology 7, 191-202. 
Downey, D.J., Simkin, P.A., Taggart, R., 1988. The effect of compressive loading on intraosseous pressure in the femoral head in vitro. The Journal of bone and joint surgery American Volume 70, 871-877.

Forgacs, G., 1995. Biological specificity and measurable physical properties of cell surface receptors and their possible role in signal transduction through the cytoskeleton. Biochem. Cell Biol. 73, 317-326.

Gilsanz, V., Wren, T.A., Sanchez, M., Dorey, F., Judex, S., Rubin, C., 2006. Low-level, highfrequency mechanical signals enhance musculoskeletal development of young women with low BMD. J. Bone Miner. Res. 21, 1464-1474.

Hu, M., Cheng, J., Qin, Y.X., 2012. Dynamic hydraulic flow stimulation on mitigation of trabecular bone loss in a rat functional disuse model. Bone 51, 819-825.

Judex, S., Lei, X., Han, D., Rubin, C., 2007. Low-magnitude mechanical signals that stimulate bone formation in the ovariectomized rat are dependent on the applied frequency but not on the strain magnitude. J. Biomech. 40, 1333-1339.

Justesen, J., Stenderup, K., Ebbesen, E.N., Mosekilde, L., Steiniche, T., Kassem, M., 2001. Adipocyte tissue volume in bone marrow is increased with aging and in patients with osteoporosis. Biogerontology 2, 165-171.

Kafka, V., 1983. On hydraulic strengthening of bones. Biorheology 20, 789-793.

Kapur, S., Baylink, D.J., Lau, K.H.W., 2002. Fluid flow shear stress stimulates human osteoblast proliferation and differentiation through multiple interacting and competing signal transduction pathways. Bone 32, 241-251.

Keaveny, T.M., Morgan, E.F., Niebur, G.L., Yeh, O.C., 2001. Biomechanics of trabecular bone. Annu. Rev. Biomed. Eng. 3, 307-333.

Keyak, J.H., Fourkas, M.G., Meagher, J.M., Skinner, H.B., 1993. Validation of an automated method of three-dimensional finite element modelling of bone. J Biomed Eng 15, 505509.

Keyak, J.H., Meagher, J.M., Skinner, H.B., Mote, C.D., Jr., 1990. Automated three-dimensional finite element modelling of bone: a new method. J. Biomed. Eng. 12, 389-397.

Kohles, S.S., Roberts, J.B., Upton, M.L., Wilson, C.G., Bonassar, L.J., Schlichting, A.L., 2001. Direct perfusion measurements of cancellous bone anisotropic permeability. J. Biomech. 34, 1197-1202.

Kumar, S., Davis, P.R., Pickles, B., 1979. Bone-marrow pressure and bone strength. Acta Orthop. Scand. 50, 507-512.

Kwon, R.Y., Meays, D.R., Meilan, A.S., Jones, J., Miramontes, R., Kardos, N., Yeh, J.C., Frangos, J.A., 2012. Skeletal Adaptation to Intramedullary Pressure-Induced Interstitial Fluid Flow Is Enhanced in Mice Subjected to Targeted Osteocyte Ablation. Plos One 7. 
LeBlanc, A.D., Schneider, V., Shackelford, L., West, S., Oganov, V., Bakulin, A., Voronin, L., 2000. Bone mineral and lean tissue loss after long duration space flight. J Musculoskelet Neuronal Interact 1, 157-160.

LeBlanc, A.D., Schneider, V.S., Evans, H.J., Engelbretson, D.A., Krebs, J.M., 1990. Bone mineral loss and recovery after 17 weeks of bed rest. J. Bone Miner. Res. 5, 843-850.

Li, Y.J., Batra, N.N., You, L., Meier, S.C., Coe, I.A., Yellowley, C.E., Jacobs, C.R., 2004. Oscillatory fluid flow affects human marrow stromal cell proliferation and differentiation. J. Orthop. Res. 22, 1283-1289.

Lotz, J.C., Cheal, E.J., Hayes, W.C., 1991. Fracture prediction for the proximal femur using finite element models: Part I-Linear analysis. J. Biomech. Eng. 113, 353-360.

Lotz, J.C., Gerhart, T.N., Hayes, W.C., 1990. Mechanical properties of trabecular bone from the proximal femur: A quantitative CT study. J. Comput. Assisted Tomogr. 14, 107-114.

Luu, Y.K., Capilla, E., Rosen, C.J., Gilsanz, V., Pessin, J.E., Judex, S., Rubin, C.T., 2009. Mechanical stimulation of mesenchymal stem cell proliferation and differentiation promotes osteogenesis while preventing dietary-induced obesity. J. Bone Miner. Res. 24, 50-61.

Lynch, M.E., Brooks, D., Mohanan, S., Lee, M.J., Polamraju, P., Dent, K., Bonassar, L.J., van der Meulen, M.C., Fischbach, C., 2013. In vivo tibial compression decreases osteolysis and tumor formation in a human metastatic breast cancer model. J. Bone Miner. Res. 28, 2357-2367.

Mantila Roosa, S.M., Liu, Y., Turner, C.H., 2011. Gene expression patterns in bone following mechanical loading. J. Bone Miner. Res. 26, 100-112.

McAllister, Du, Frangos, 2000. Fluid shear stress stimulates prostaglandin and nitric oxide release in bone marrow-derived preosteoclast-like cells. Biochem. Biophys. Res. Commun. 270, 643-648.

Metzger, T.A., Kreipke, T.C., Vaughan, T.J., McNamara, L.M., Niebur, G.L., 2015. The In Situ Mechanics of Trabecular Bone Marrow: The Potential for Mechanobiological Response. J. Biomech. Eng. 137.

Metzger, T.A., Shudick, J.M., Seekell, R., Zhu, Y., Niebur, G.L., 2014. Rheological behavior of fresh bone marrow and the effects of storage. J Mech Behav Biomed Mater 40, 307-313.

Nauman, E.A., Fong, K.E., Keaveny, T.M., 1999. Dependence of inter-trabecular permeability on flow direction and anatomic site. Ann. Biomed. Eng. 27, 517-524.

Naveiras, O., Nardi, V., Wenzel, P.L., Hauschka, P.V., Fahey, F., Daley, G.Q., 2009. Bonemarrow adipocytes as negative regulators of the haematopoietic microenvironment. Nature 460, 259-263. 
418 Ochoa, J.A., Hillberry, B.M., Year A poroelastic model for the hydraulic stiffening of cancellous bone. In Trans. Orthop. Res. Soc. Washington, D.C.

Ochoa, J.A., Sanders, A.P., Heck, D.A., Hillberry, B.M., 1991. Stiffening of the femoral head due to inter-trabecular fluid and intraosseous pressure. J. Biomech. Eng. 113, 259-262.

Ochoa, J.A., Sanders, A.P., Kiesler, T.W., Heck, D.A., Toombs, J.P., Brandt, K.D., Hillberry, B.M., 1997. In vivo observations of hydraulic stiffening in the canine femoral head. J. Biomech. Eng. 119, 103-108.

Ozcivici, E., Luu, Y.K., Adler, B., Qin, Y.-X., Rubin, J., Judex, S., Rubin, C.T., 2010. Mechanical signals as anabolic agents in bone. Nature Reviews Rheumatology 6, 50-59.

Preziosi, L., Ambrosi, D., Verdier, C., 2010. An elasto-visco-plastic model of cell aggregates. J. Theor. Biol. 262, 35-47.

Qin, Y., 2003. Fluid pressure gradients, arising from oscillations in intramedullary pressure, is correlated with the formation of bone and inhibition of intracortical porosity. J. Biomech. 36, 1427-1437.

Rosen, C.J., Bouxsein, M.L., 2006. Mechanisms of disease: is osteoporosis the obesity of bone? Nat Clin Pract Rheumatol 2, 35-43.

Rutgers, I.R., 1962. Relative viscosity of suspensions of rigid spheres in Newtonian liquids. Rheologica Acta 2, 202-210.

Schaffler, M.B., Cheung, W.Y., Majeska, R., Kennedy, O., 2014. Osteocytes: master orchestrators of bone. Calcif. Tissue Int. 94, 5-24.

Soves, C.P., Miller, J.D., Begun, D.L., Taichman, R.S., Hankenson, K.D., Goldstein, S.A., 2014. Megakaryocytes are mechanically responsive and influence osteoblast proliferation and differentiation. Bone 66C, 111-120.

Stevens, H.Y., Meays, D.R., Frangos, J.A., 2006a. Pressure gradients and transport in the murine femur upon hindlimb suspension. Bone 39, 565-572.

Stevens, H.Y., Meays, D.R., Yeh, J., Bjursten, L.M., Frangos, J.A., 2006b. COX-2 is necessary for venous ligation-mediated bone adaptation in mice. Bone 38, 93-104.

Tavassoli, M., Yoffey, J.M., 1983. Marrow structure. Alan R. Liss, Inc., New York, NY.

Tournaire, N., Jaffre, C., Jacob, M., Ducher, G., Benhamou, C., Courteix, D., Meddahi-Pelle, A., 2007. MMP2 and MMP9 plasma levels as markers of bone remodeling: a study on young male tennis players. Science \& sports 22, 123-125.

Vaughan, T.J., Voisin, M., Niebur, G.L., McNamara, L.M., 2015. Multiscale Modeling of Trabecular Bone Marrow: Understanding the Micromechanical Environment of Mesenchymal Stem Cells During Osteoporosis. J. Biomech. Eng. 137.

Viceconti, M., Toni, A., Giunti, A., 1992. Strain gauge analysis of hard tissues: factors influencing measurements. Elsevier Science, Amsterdam. 
454 Webster, D., Schulte, F.A., Lambers, F.M., Kuhn, G., Muller, R., 2015. Strain energy density gradients in bone marrow predict osteoblast and osteoclast activity: A finite element study. J. Biomech. 48, 866-874.

457 Welch, R.D., Johnston, C.E., 2nd, Waldron, M.J., Poteet, B., 1993. Bone changes associated with intraosseous hypertension in the caprine tibia. J Bone Joint Surg Am 75, 53-60.

459 Xie, L., Jacobson, J.M., Choi, E.S., Busa, B., Donahue, L.R., Miller, L.M., Rubin, C.T., Judex,

460 S., 2006. Low-level mechanical vibrations can influence bone resorption and bone

461 formation in the growing skeleton. Bone 39, 1059-1066.

462 You, L.D., Temiyasathit, S., Lee, P.L., Kim, C.H., Tummala, P., Yao, W., Kingery, W., Malone,

463 A.M., Kwon, R.Y., Jacobs, C.R., 2008. Osteocytes as mechanosensors in the inhibition of 464 bone resorption due to mechanical loading. Bone 42, 172-179.

465 Yourek, G., McCormick, S.M., Mao, J.J., Reilly, G.C., 2010. Shear stress induces osteogenic 466 differentiation of human mesenchymal stem cells. Regen Med 5, 713-724. 


\section{Table Captions}

469 Table 1. Average of the absolute value of the measured pressure gradients over the first $5 \mathrm{sec}$ of

470 loading for the stress-relaxation specimens and over the first, full $1 \mathrm{sec}$ cycle of the cyclically

471 loaded specimens. Proximal represents the gradients between adjacent transducers closest to the

472 femoral head (PT1-PT2) and distal represents the gradient between adjacent transducers most

473 distal to femoral head (PT3-PT4).

474 Table 2. The orthotropic intrinsic permeability was determined from image-based fluid flow

475 models of $4 \times 4 \times 4 \mathrm{~mm}$ trabecular bone images from five of the cyclically loaded femurs, and

476 applied to continuum poroelastic analyses. Samples span the entire range of permeability

477 measured across all specimens studied.

478 Table 3. Measured mechanostimulatory shear stress thresholds for resident marrow cell

479 populations found in literature. 


\section{Figure Captions}

Figure 1: A) Four holes were drilled along the femoral neck to provide insertion points for miniature pressure transducers. Inset - Transducers were fed through cannulae, which were press-fit into the holes (scale bar $=5 \mathrm{~mm}$ ). One arm of the cannula was used to pass the transducer leads, while the other was used to backfill the hole with water. Both were sealed with epoxy. Strain gauge rosettes were attached to the anterior and posterior greater trochanter. B) The femora were tested while submerged in a water bath. The distal end of the femur was potted in PMMA and fixed to the water filled tank. A circulating heater was used to maintain the water at $37^{\circ} \mathrm{C}$ throughout testing.

Figure 2: A) Femora were imaged using micro-CT at locations between pressure transducers at a resolution of $20 \mu \mathrm{m}$. B) A $4 \mathrm{~mm}^{3}$ cubic region was selected from between adjacent pressure transducers and C) the marrow space was meshed with approximately 1 million tetrahedral finite elements. The measured pressure differentials were applied to the proximal face of models with zero pressure applied to the distal face. Inset - A no-slip boundary condition was applied on the lateral faces of the fluid models. D) Computational fluid dynamics models were solved to find the marrow shear stress, which is depicted on streamlines in this image.

Figure 3: A) The orthotropic permeability was determined for five porcine specimens by applying a pressure gradient along each coordinate axis, with $80 \mathrm{~Pa}$ applied on one face of the fluid and $0 \mathrm{~Pa}$ on the opposing face. A thin layer of fluid surrounded the entire model to allow

501 uniform boundary conditions (not shown for clarity). B) The Darcy permeability tensors were 502 calculated as the ratio of flow-rate to the calculated pressure gradient along all three coordinate axes. Five finite element models with one thousand hexahedral elements were created with

504 orthotropic permeability corresponding to the principal values from the tensors calculated in each 505 micro-scale model, a uniform isotropic modulus of $500 \mathrm{MPa}$, and Poisson's ratio of 0.3

506 (Keaveny et al., 2001). The models were solved for boundary conditions where the fluid was 507 constrained to only flow through the superior surface (C and D) or through the superior and 508 lateral surfaces (E and F).

510 Figure 4: A) Bone marrow pressure increased when a static load was applied. The average 511 peak pressure was $1.7 \pm 1.3 \mathrm{kPa}$ (mean \pm std). Pressure dissipated during the holding period, but 512 had little effect on the applied load. The strain rate on the anterior cortex was approximately

$5130.02 \% \mathrm{~s}^{-1}$. B) The pressure varied sinusoidally with load during cyclic loading, with an average 514 pressure differential of $0.73 \pm 0.57 \mathrm{kPa}$ during the first full loading cycle across all of the 
515 specimens. The strain rate on the anterior cortex was approximately $0.12 \% \mathrm{~s}^{-1}$ during cyclic

516 loading. Representative sample depicted.

517

518 Figure 5: Time-averaged pressure gradients were calculated with transducer spacing determined 519 from the micro-CT images. Pressure gradients did not correlate to trabecular bone volume 520 fraction during A) stress-relaxation or B) cyclic loading. Each color represents the three samples 521 from a single femur.

522

523 Figure 6: A) Constrained and B) free-flow boundary conditions were applied to continuum 524 poroelastic analysis under five loading conditions. Fluid flow was restricted to one face of the 525 model in constrained models, while fluid flow was unrestricted to all faces except one in the 526 free-flow model. Pore pressure gradients decreased with increasing intrinsic permeability. The 527 permeability in the z-direction was chosen for each of the models. Markers represent time528 averaged pressure gradients measured experimentally during cyclic loading, with each color 529 representing one femur.

531 Figure 7: A) The volumetric- and time-averaged mean shear stress ranged from 0.311 to 16.42

$532 \mathrm{~Pa}$ during stress-relaxation and increased with the time-averaged pressure gradient. B) The 533 volumetric- and time-averaged shear stress during cyclic loading ranged from 2.14 to $17.57 \mathrm{~Pa}$ 534 and increased with the time-averaged pressure gradient. Each color represents the three samples 535 from a single femur. Dashed lines are $\pm 95 \%$ C.I. 
Figure 1

\section{A}

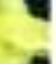

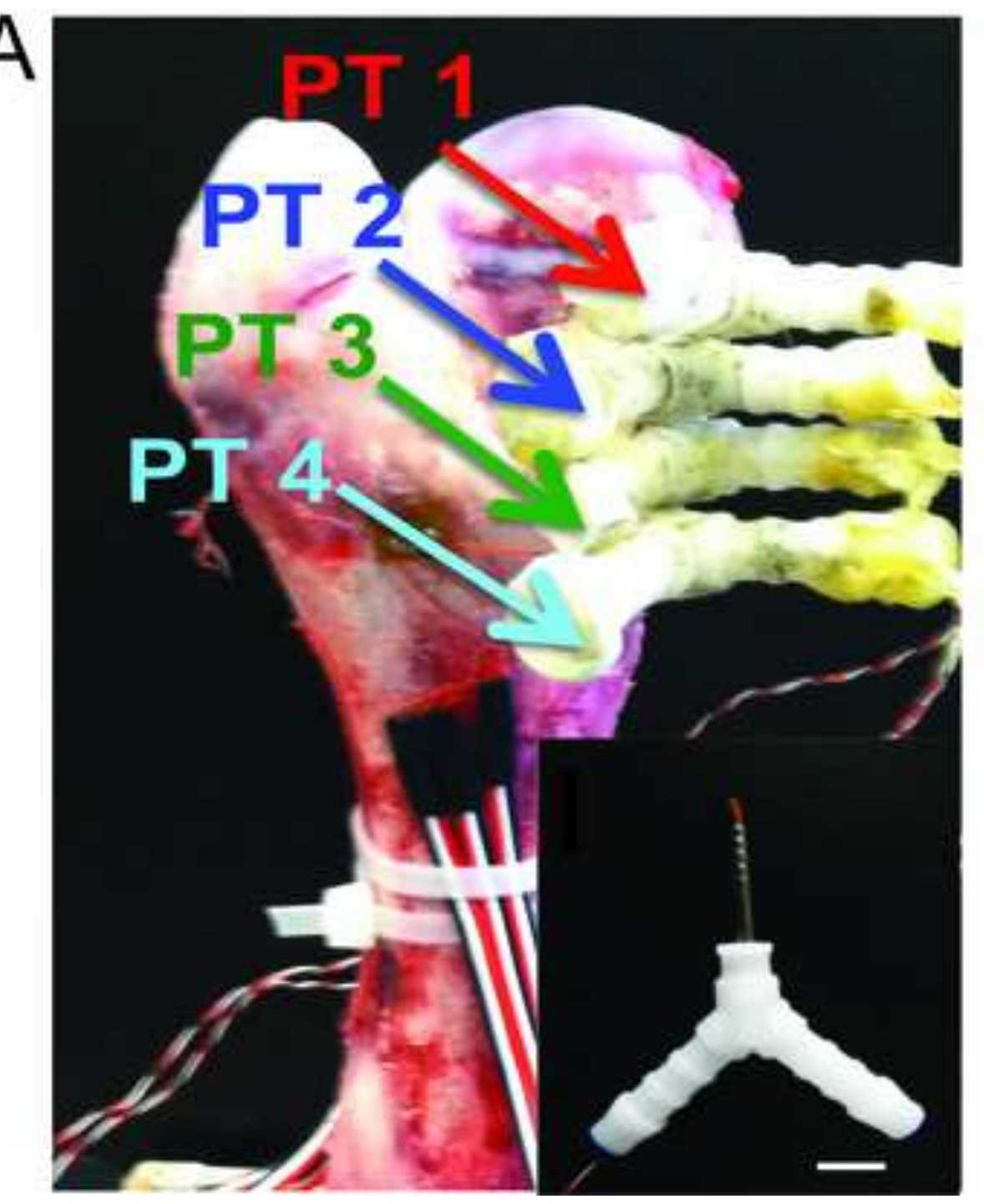

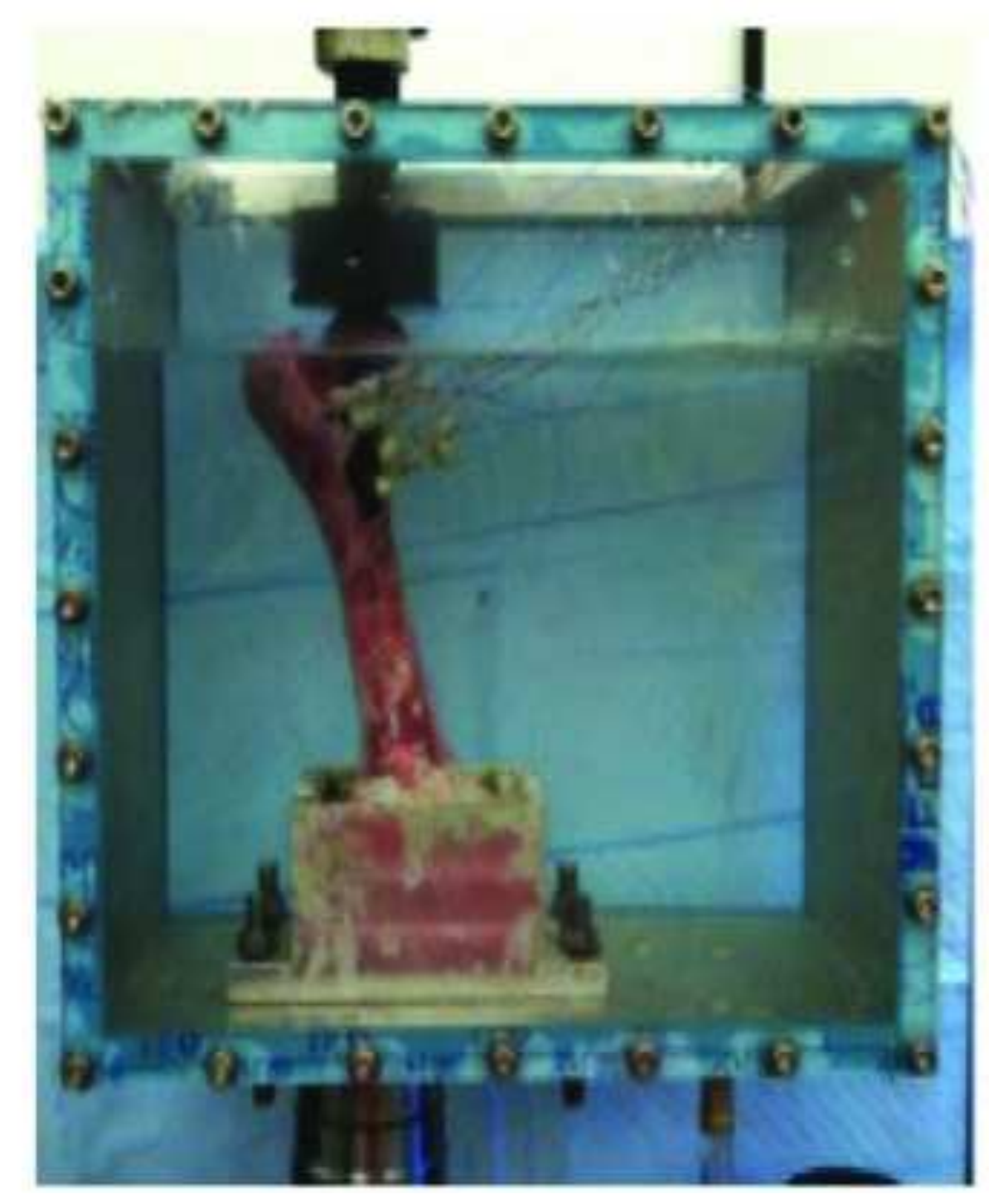

\section{B} 4

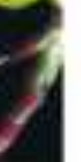
. 
A
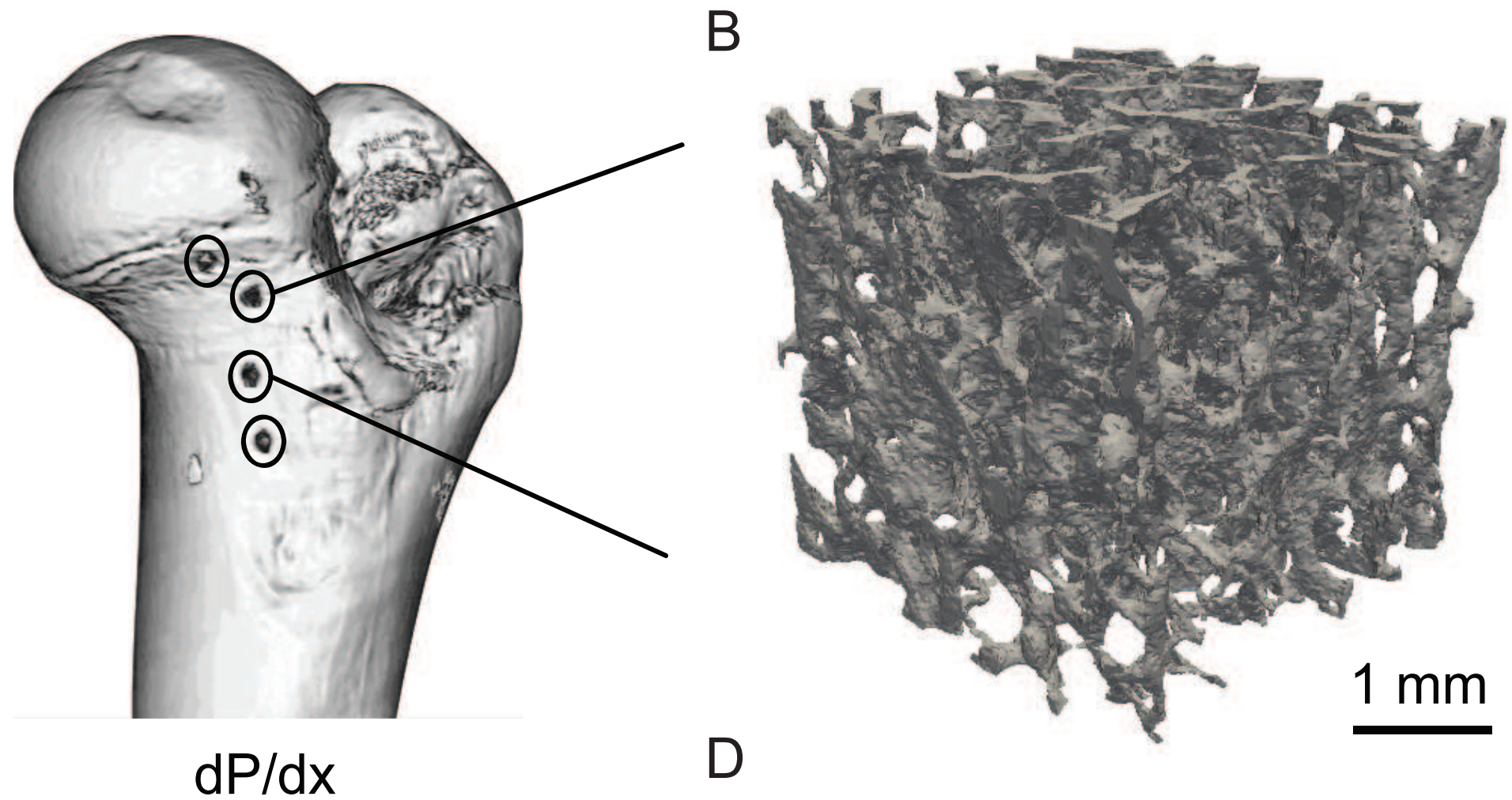

C

D

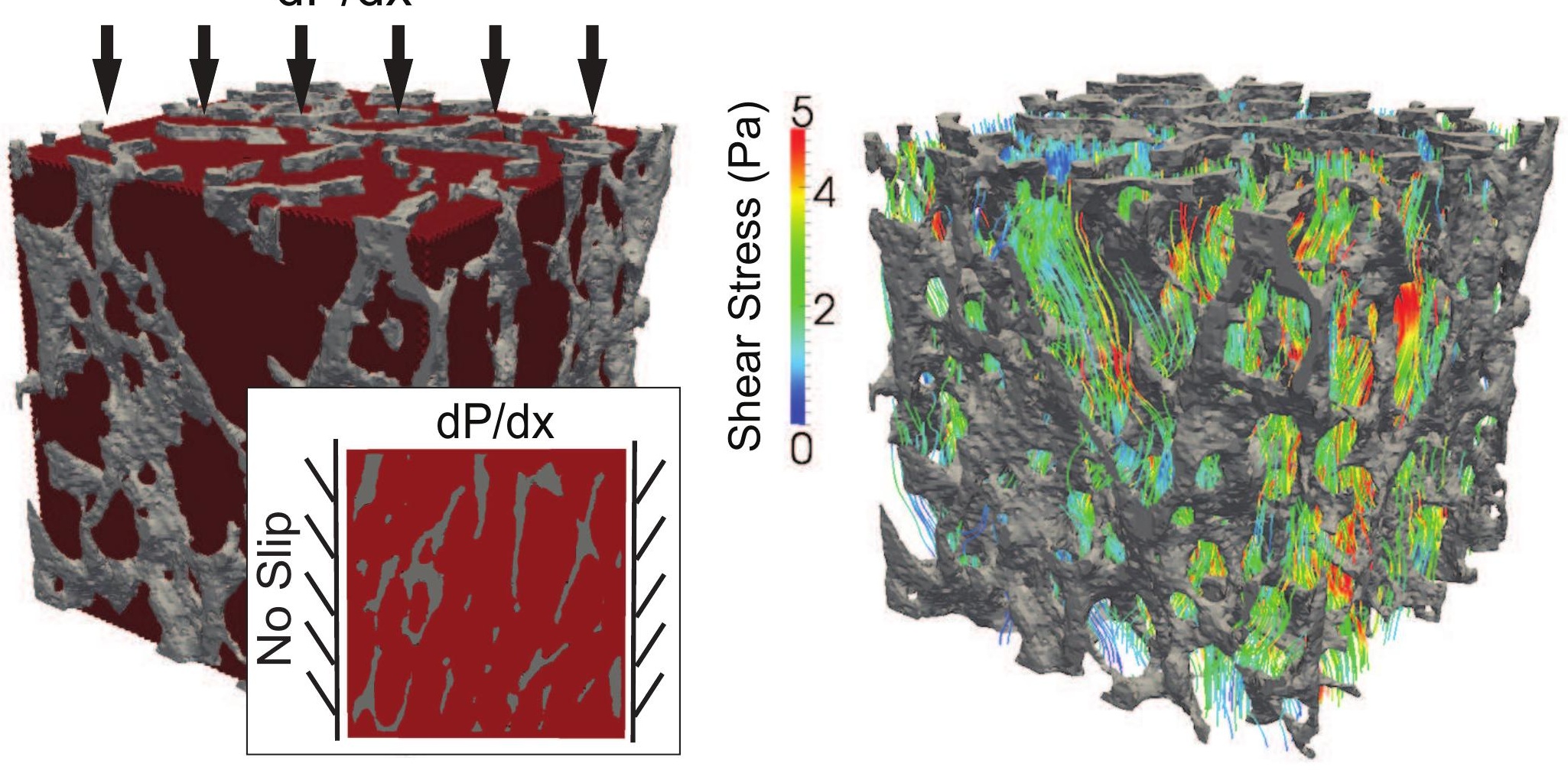


Figure 5

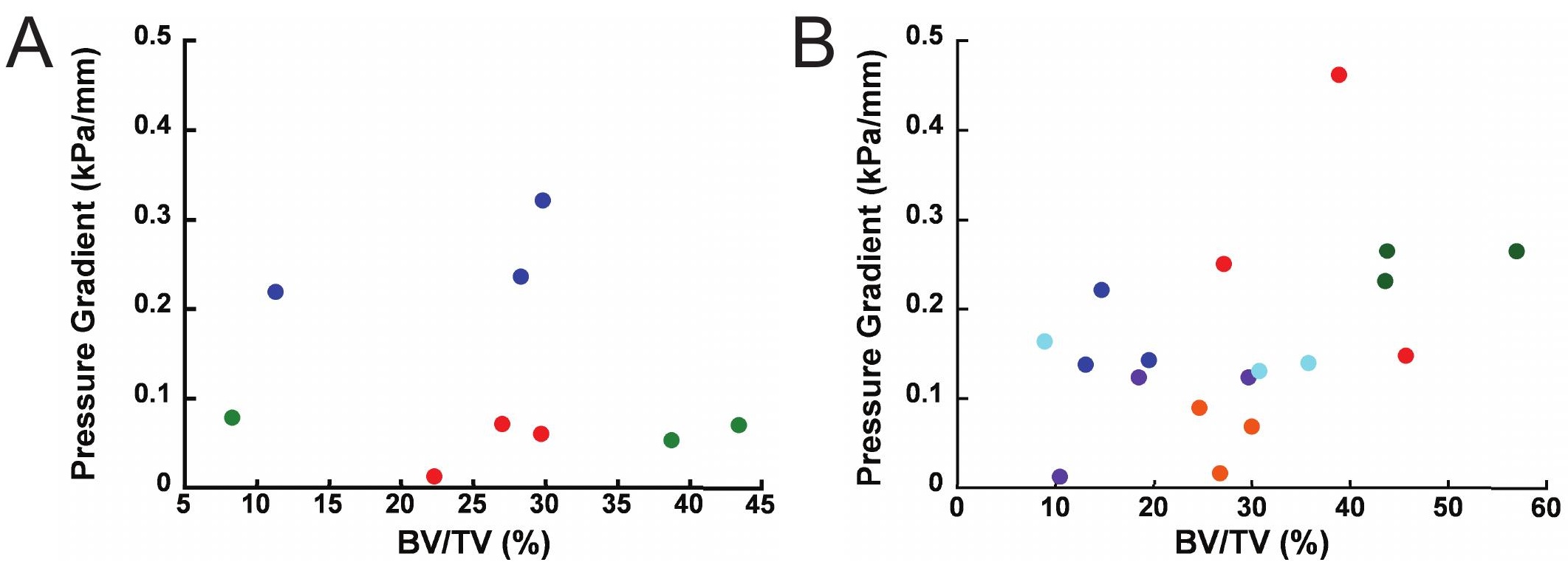


Figure 6

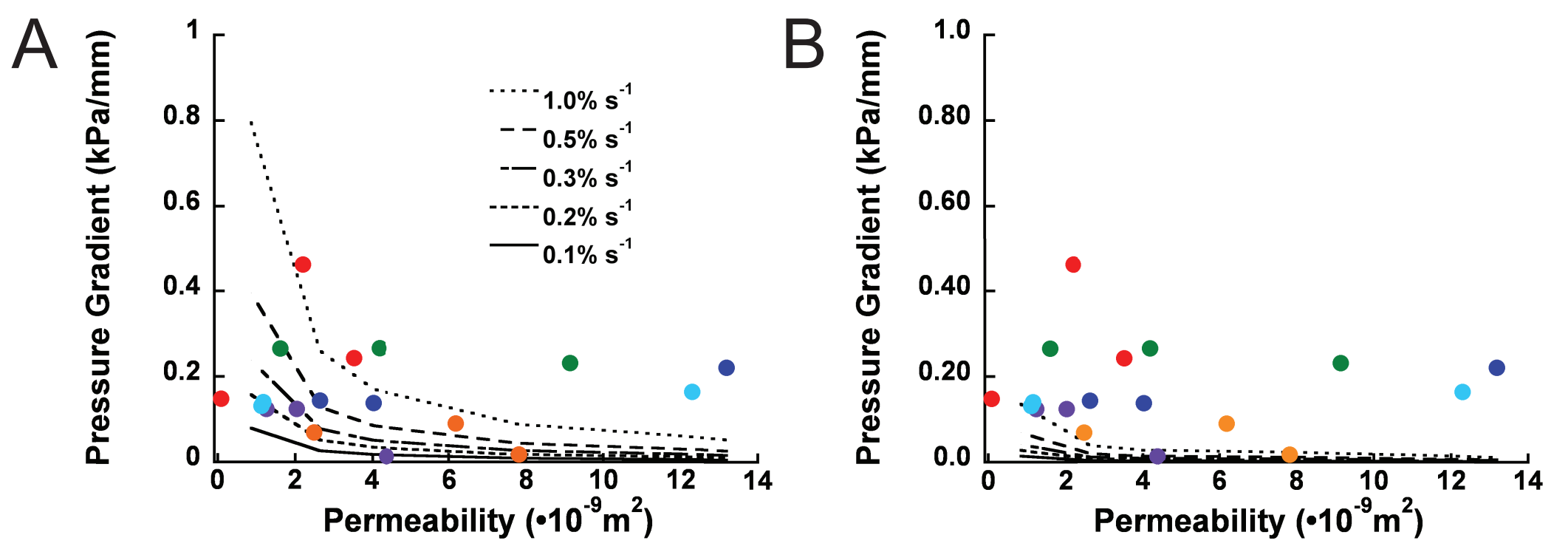


Stress-Relaxation

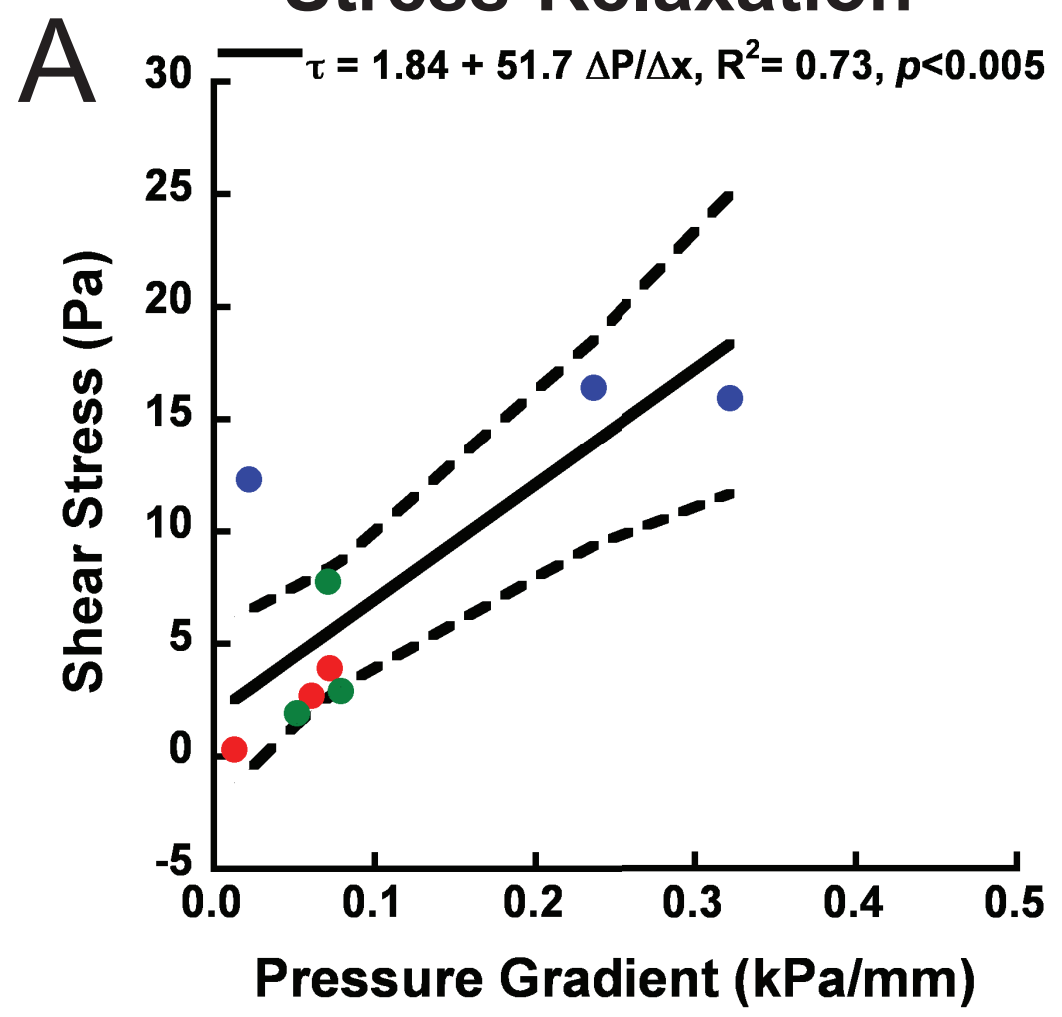

Cyclic

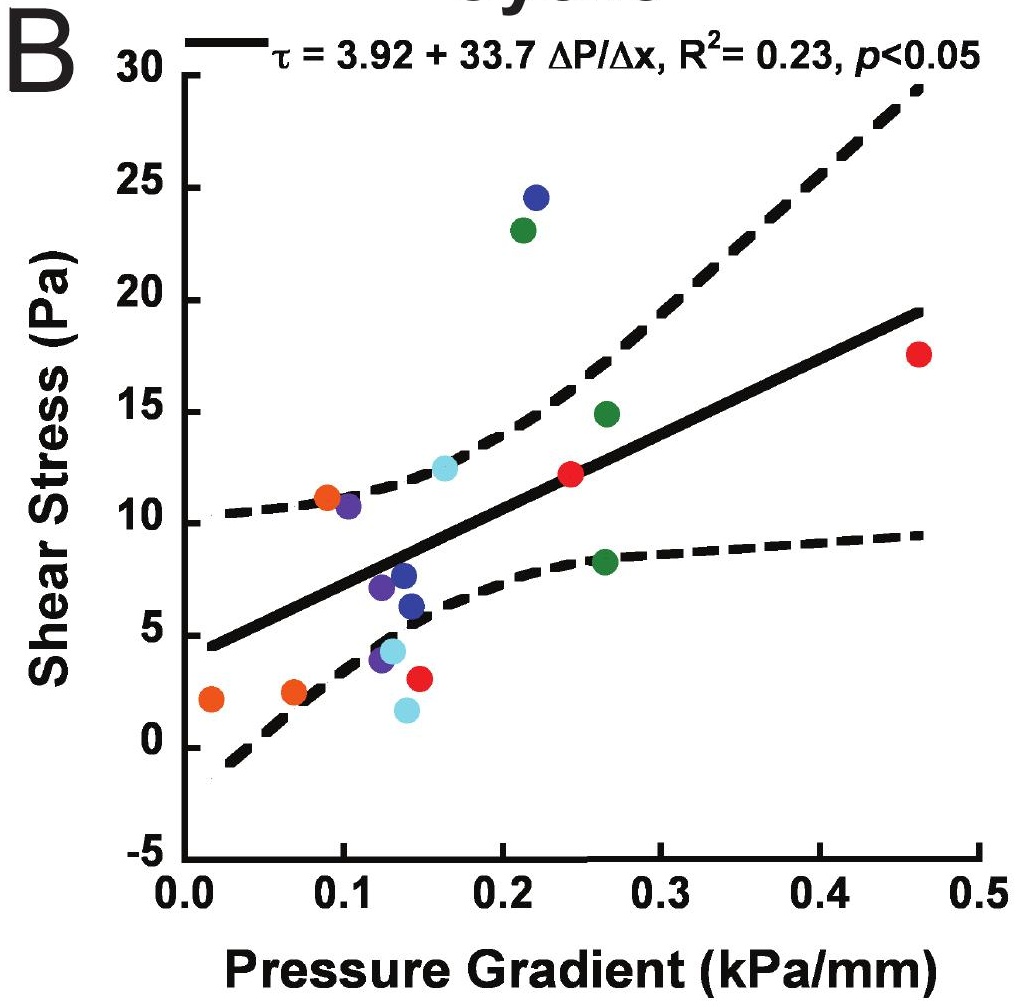


Table 1. Average of the absolute value of the measured pressure gradients over the first $5 \mathrm{sec}$ of loading for the stress-relaxation specimens and over the first, full $1 \mathrm{sec}$ cycle of the cyclically loaded specimens. Proximal represents the gradients between adjacent transducers closest to the femoral head (PT1-PT2) and distal represents the gradient between adjacent transducers most distal to femoral head (PT3-PT4).

\begin{tabular}{lccc}
\hline & \multicolumn{3}{c}{ Pressure Gradient (kPa/mm) } \\
& Proximal & Middle & Distal \\
\hline Stress-Relaxation & & & \\
Specimen 1 & 0.013 & 0.061 & 0.072 \\
Specimen 2 & 0.052 & 0.079 & 0.071 \\
Specimen 3 & 0.322 & 0.236 & 0.219 \\
Cyclic & & & \\
Specimen 1 & 0.124 & 0.124 & 0.103 \\
Specimen 2 & 0.069 & 0.090 & 0.017 \\
Specimen 3 & 0.143 & 0.138 & 0.221 \\
Specimen 4 & 0.265 & 0.266 & 0.213 \\
Specimen 5 & 0.148 & 0.462 & 0.243 \\
Specimen 6 & 0.131 & 0.140 & 0.164 \\
\hline
\end{tabular}

Table 2. The orthotropic intrinsic permeability was determined from image-based fluid flow models of $4 \times 4 \times 4 \mathrm{~mm}$ trabecular bone images from five of the cyclically loaded femurs, and applied to continuum poroelastic analyses. Samples span the entire range of permeabilities measured across all porcine bone specimens studied.

\begin{tabular}{lccc}
\hline & & Permeability $\left(\mathbf{m}^{2}\right)$ & \\
& Medial-Lateral & Anterior-Posterior & Proximal-Distal \\
\hline Sample 1 & $4.77 \cdot 10^{-10}$ & $2.98 \cdot 10^{-10}$ & $8.64 \cdot 10^{-10}$ \\
Sample 2 & $1.17 \cdot 10^{-9}$ & $1.65 \cdot 10^{-9}$ & $2.64 \cdot 10^{-9}$ \\
Sample 3 & $1.32 \cdot 10^{-9}$ & $2.56 \cdot 10^{-9}$ & $4.03 \cdot 10^{-9}$ \\
Sample 4 & $3.36 \cdot 10^{-9}$ & $8.35 \cdot 10^{-9}$ & $7.82 \cdot 10^{-9}$ \\
Sample 5 & $5.61 \cdot 10^{-9}$ & $3.11 \cdot 10^{-9}$ & $1.32 \cdot 10^{-8}$ \\
\hline
\end{tabular}

Table 3. Measured mechanostimulatory shear stress thresholds for resident marrow cell populations found in literature.

\begin{tabular}{ccc}
\hline & Threshold (Pa) & Source \\
\hline Mesenchymal stem cells & 0.90 & (Yourek et al., 2010) \\
Osteoblasts & 2.0 & (Kapur et al., 2002) \\
Osteoclasts & $0.5-2.0$ & (Cui et al., 2012) \\
Megakaryocytes & $1-2$ & (Soves et al., 2014) \\
\hline
\end{tabular}

